\title{
Reflections on Physics Education and Communication with Tibetan Monastics
}

\author{
Judith Beck ${ }^{1}$, Justin C. Burton ${ }^{2 *}$, Martin Kamela ${ }^{3}$, Michelle P. Kuchera ${ }^{4}$, Amy J. Lovell ${ }^{5}$, \\ Kerstin Nordstrom ${ }^{6}$, Thinley Tenzin ${ }^{7}$ and Julie Ziffer ${ }^{8}$ \\ ${ }^{1}$ Department of Physics and Astronomy, University of North Carolina Asheville, Asheville, NC, United States, ${ }^{2}$ Department of \\ Physics, Emory University, Atlanta, GA, United States, ${ }^{3}$ Department of Physics, Elon University, Elon, NC, United States, \\ ${ }^{4}$ Department of Physics, Davidson College, Davidson, NC, United States, ${ }^{5}$ Department of Physics and Astronomy, Agnes Scott \\ College, Decatur, GA, United States, ${ }^{6}$ Department of Physics, Mount Holyoke College, South Hadley, MA, United States, ${ }^{7}$ Sera \\ Jey Monastic University, Bylakuppe, India, ${ }^{8}$ Department of Physics, University of Southern Maine, Portland, ME, United States
}

OPEN ACCESS

Edited by:

Robin Nusslock, Northwestern University,

United States

Reviewed by:

Stamatis Vokos,

California Polytechnic State University,

United States

Rabindra Bajracharya, Missouri Southern State University,

United States

*Correspondence:

Justin C. Burton

justin.c.burton@emory.edu

Specialty section:

This article was submitted to

Science and Environmental

Communication,

a section of the journal

Frontiers in Communication

Received: 26 June 2021 Accepted: 12 October 2021

Published: 02 November 2021

Citation:

Beck J, Burton JC, Kamela M, Kuchera MP, Lovell AJ, Nordstrom K, Tenzin T and Ziffer J (2021) Reflections

on Physics Education and

Communication with

Tibetan Monastics.

Front. Commun. 6:731074.

do: $10.3389 /$ fcomm.2021.731074
Physics education research, a field that is now over 100 years old, has made it clear: the key to teaching physics successfully is to prioritize getting the concepts across, and the mathematical representation ("laws") can come later. On the other hand, conceptual representations are steeped in culture and language, while the corresponding "laws" are universal. The ETSI program provided a unique opportunity to re-imagine conceptual physics for Tibetan monastic culture. This perspective describes the major challenges derived from this experience over multiple areas of physics, and addresses two central questions: 1) how does the translation and interpretation of key terms such as energy, motion, charge, and system change the connotations surrounding physical laws? 2) What are the practical strengths and weaknesses of teaching conceptual physics using welldeveloped methods in the United States, and what can educators learn about the emergence of understanding?

Keywords: physics education, Tibet, physics demonstration, monastic culture, science education

\section{INTRODUCTION}

From 2008 to 2013, the Emory-Tibet Science Initiative (ETSI) ETSI (2021) sponsored 36 science faculty, mostly from Emory University, to visit Dharamshala, India and deliver short 4-5 weeks courses in philosophy of science, physics, neuroscience, and biology. Building on the success of this program, from 2014 to 2019, ETSI expanded into its Implementation Phase by introducing a 6-year curriculum in these subjects, thereby establishing science education as part of the core curriculum of three major Tibetan monastic universities in India. Enrollment in the program grew from 345 in 2014 to 1,496 in 2019. By the end of the Implementation Phase, each university required two physics faculty for each year of study (Years 1-6). The faculty were recruited from a broad range of universities and colleges, primarily in the United States of America (United States). This perspective serves as a reflection of this experience, the communication challenges, and the major co-learned takeaways from a meshing of United States physics education with Tibetan Buddhist philosophy.

Throughout this article we use the term scholars to refer to the monastic students, both monks and nuns. The physics curriculum consisted of a selection of core concepts with a different focus each year: an introduction to the study of physics (Year 1), motion and mechanics (Year 2), thermodynamics and waves (Year 3), electricity and magnetism, and light, (Year 4), optics and modern physics (Year 5), and astronomy (Year 6). The primary reference was a Tibetan translation 

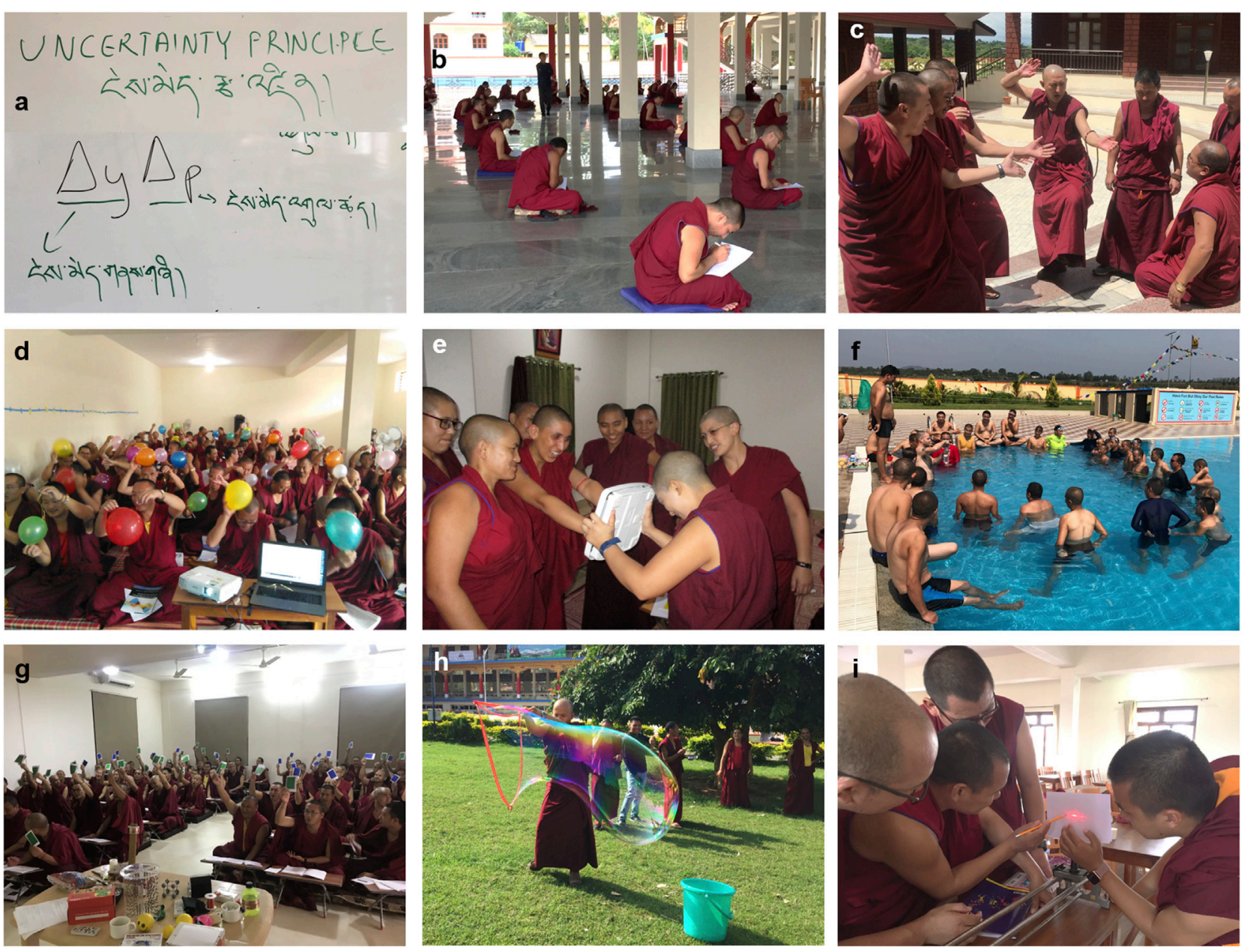

FIGURE 1 | (A) Tibetan translations of physics terms. (B) At the end of 2-weeks course, a written, translated exam for each year of the program assessed the course. (C) Debates occurred nearly every evening, a key component of monastic education. (D) Scholars learn about static electricity with balloons. (E) Female year 2 scholars experimenting with forces as indicated on a scale. (F) An outdoor class on buoyancy in the swimming pool during Year 3. (G) Scholars used colored cards to answer physics concept questions. (H) Making large bubbles to highlight thin film interference of light. (G) Year 5 experiments included measuring diffraction with a laser pointer.

of a college-level physics textbook, Conceptual Physics Hewitt (2010). The ordering and choice of topics, as well as the choice of reference text, were all predetermined before the implementation phase. Thus faculty were charged with implementing this specific curriculum, but had freedom to choose instructional methods.

Typically, two translators were assigned to each class. Translators and faculty would meet to review the upcoming material (Figures 1A,B), yet most translation was done in real-time and was challenging for some concepts. For less experienced science translators, we adopted a nearly sentenceby-sentence method. A translator with more physics experience could present ideas more fully, serving more as a co-teacher and preventing some terminology misunderstanding and confusion.

Most physics faculty in the program (and all authors on this paper), chose active learning techniques typical of a modern undergraduate course in the United States. It has been demonstrated for United States undergraduates that physics learning happens most readily where students are actively engaged in course topics, via hands-on activities and peer-topeer discussions Hake (1998); Meltzer and Thornton (2012). The active engagement aimed for in the physics classes was not dissimilar, in its goal, to some of the practices of Buddhist monastics, the most notable example is an exercise translated into English as "debating" (Dreyfus (2003)).

Debating involves two individuals or teams arguing opposing sides of a question, by drawing on knowledge and applying critical reasoning and logic. However, debates at the monastery bear little outward resemblance to formal debate as practiced in the United States. These are loud, physically active debates in an outdoor courtyard, with monks shouting over each other and slapping their hands together to relish a momentary victory or well-made point (Figure 1C). Intensive training in debate appeared to influence the way the scholars approached learning. Questioning was an important and celebrated part of the learning process. Since scholars relished challenging assumptions and probing arguments for weaknesses, we tried incorporating 
debate into our physics classes. For example, by Year 5, scholars had been exposed to many historical experiments and observations that fundamentally changed our understanding of the natural world. For example, one question posed for debate was: Is experimentation necessary for the advancement of science?

The remaining sections of this article focus specifically on the content and collective experience of the authors for each Year of study. Attention is paid to misunderstandings of foundational principles and learning styles that are distinct from those often found in undergraduate classes, although similarities will be discussed as well.

\section{YEARS 1 AND 2}

The Year 1 ETSI physics curriculum gave an overview of the field, introduced a wide array of topics and built excitement about the modules to come. Since mathematics is wholly integrated into the language of physics, the first course was also a place to identify and build quantitative skills that were important for engagement in future material. The Year 2 Mechanics course focused on motion, forces, and energy, and began to put these math skills to work. While most scholars found the operations of addition and subtraction to be intuitive, the physics curriculum also required facility with less-intuitive concepts such as negative quantities, powers and exponents, and direct and inverse proportionality.

Scholars were fascinated by more modern and advanced topics, such as special and general relativity and quantum systems. A significant communications challenge in the early years of the curriculum was connecting fundamentals to these more exciting topics where possible and emphasizing the connections across the field. This approach is consistent with best practices of physics education in the United States, aiming for a coherent view of the physical world across a wide range of phenomena Redish (2003).

In physics, terms like energy, momentum, displacement, etc., are used to describe motion and are specific in their definitions. These terms also describe specific ways for making sense of objects in motion. When translating these terms there are two challenges to overcome: 1) there may not be a word in the Tibetan language that corresponds closely to the physics term in English, and 2) the way motion is conceptualized in the Tibetan Buddhist philosophy may not map easily to how physicists think of motion.

In a survey conducted after the 2019 Mechanics Year 2 module, scholars were asked "When you kick a ball off the ground, why does the ball continue to move after it left your foot?". Physicists would answer the question with the concept of inertia, and with momentum and energy, imparted to the ball by the foot, that remain with the ball in flight. A typical response by the monastic scholars was to acknowledge inertia, but also focus on the notion of impetus, that the "kick force remains continuously to make (the ball) move". This is not dissimilar to typical introductory physics classrooms in the United States, but Year 2 scholars may have also been grappling with the complex notion of causality in Tibetan Buddhist philosophy in addition to making sense of physical motion Kalupahana (1975).
For physicists a force is an action that, in the case of the kicked ball, is short in duration. The momentum and energy of the ball are properties of the object affected by forces. In the Tibetan language, energy and force may be used interchangeably, presenting a translation difficulty that exemplifies the challenge of communicating and translating in real time without preparation. Cultural conceptions of the world also need to be explicitly taken into consideration to make the translation meaningful Aikenhead and Jegede (1999); Redish (2012). Similarly, in the undergraduate physics classroom, acknowledging students' current conception of motion before proceeding to explore the physicists' consensus on the topic is recognized as a best practice Novak et al., 1999.

It is increasingly common in undergraduate science teaching to take a hands-on, experiential approach that connects theoretical concepts to practical applications Hake (1998). Wherever possible within the constraints of available materials, we integrated hands-on activities to help build intuition about physical systems. With an activity, students can ask and answer questions arising from basic curiosity, and immediately test predictions. For example, electric charge is not a visible quantity, but the scholars could build up charge on the surface of a balloon, and experience the results (Figure 1D). Wheeled carts helped scholars experience motion and relate, for example, force and acceleration. Scholars pressed on a scale to gain intuition with applied forces and reaction forces (Figure 1E). With practical activities, scholars could pose and answer questions in a learning-focused environment, helping overcome potential barriers to questioning a teacher perceived to be an expert.

\section{YEARS 3 AND 4}

The Year 3 curriculum introduced the scholars to properties of matter, building blocks such as atoms, molecules, chemicals, and the periodic table. One major piece of introductory material was understanding large numbers. Questions such as "How many molecules are there in a water drop?" led to philosophical discussions of breaking matter into constitutive parts, and how to represent large numbers with scientific notation, an utterly foreign concept that is central to United States curricula. Concepts such as heat, temperature, and pressure laid the foundation of thermodynamics. Energy was a common thread that ran through Year 3's discussion. Although energy is more intuitive since it represents a quantity that can change forms, there were some foundational misunderstandings, for instance, colder temperatures represent a lack of energy. Touching a cold object makes your skin cold, suggesting that something from the cold object has transferred to your skin, which is incorrect.

Another misunderstanding centered on geometrical concepts such as area and volume, especially when discussing pressure and density. This was illuminated during lessons on buoyancy. During one lesson, scholars visited the nearby swimming pool to do laboratory experiments on sinking and floating, as shown in Figure 1F. Prior to this, nearly all agreed that a heavier person, as measured by their mass, would sink in the water, and a smaller, 
lighter person would float. This myth was dispelled by direct experimentation in the pool with scholars, and by placing marbles and plastic balls in sealed water bottles.

The last third of the Year 3 curriculum focused on waves: sound, light, water, and the transfer of energy. Hands-on demonstrations included standard stretched "slinkys" and ropes, yet discussion surrounding more familiar topics such as earthquakes, musical instruments, and religious chanting always received more attention. Conservation of energy was a cornerstone concept that helped tie together thermodynamics and waves, for example, light from the Sun heats up the Earth.

The year 4 curriculum covered the topics of electricity, magnetism, and light. The mornings consisted of lecture/ discussion, and the afternoons were devoted to labs in small groups. Many of the labs involved the use of electricity and magnetism kits Chabay and Sherwood (2018) which had items such as light bulbs, switches, compasses, and magnets. While the scholars enjoyed building motors and understanding circuits, most topics in Year 4 have little payoff in terms of the bigger ideas that excite them, such as quantum physics and cosmology. This was a weakness of maintaining our familiar teaching sequence, as discussed further in the summary section.

Translation affected interpretation in some contexts, such as a circuit "switch," which was translated as "gate." This small difference led to an opposite interpretation of whether current flows when "open" and "closed". Additionally, observational evidence provided better explanation and insight than mathematical definitions. For instance, scholars constructed circuits that directly connected voltage with number of batteries, and current with light bulb brightness. This made Ohm's Law, an abstract equation, become obvious. In the case of "color," the translation was the same as "color" in Tibetan. While this allowed the scholars to identify the topic, the concept of color in Buddhist philosophy is inherently different from the way physicists conceive it Black is a color in Buddhism, whereas in the physical definition of light, black is the absence of light. Therefore, the monastics had to separate these two definitions of the same word.

A well-tested method of enabling conceptual understanding in the American classroom is to use Conceptests, also sometimes called “Think Pair Share" questions Mazur (1997); French and Prather (2018). The scholars were asked a multiple choice question, and polled for their own answer (Figure 1G). They then discussed their choice with a neighbor, and the class was repolled before the correct answer was revealed. American students are initially hesitant to participate, and some students are often easily convinced by confident neighbors. However, the monastic scholars embraced this method, and were accepting of conflict with their peers, likely due to the focus on debate and interactive discussion in monastic education.

\section{YEARS 5 AND 6}

Years 5 and 6 included three main topics: optics, modern physics and astronomy. By these years, the scholars benefited from having a substantial base of science knowledge. As a result, scholars could practice generalizing previous knowledge and applying it to novel situations. Equally importantly, scholars were now well acquainted with scientific ways of knowing and our pedagogical stylistic choices were more familiar. This allowed for smoother transitions to experiential learning and for less need to signal and support the direction the science was taking. The class lectures involved concise, distilled concepts optimized for ease of translation. This precision became particularly important when covering inherently challenging topics such as waveparticle duality and the Heisenberg Uncertainty Principle. Questions posed to the scholars were meant to generate discussion and explore misconceptions.

Optics is naturally visual, and the ability to support concept development with photos, videos, demonstrations, and labs helped engage the scholars and likely minimized some of the translation issues inherent in other parts of the curriculum. Scholars participated in investigations of visible light with polarizers, mirrors, lenses, soap bubbles, and pin-hole cameras they made out of cardboard boxes (Figures 1H,I). Ultraviolet sensitive beads and an infrared camera provided opportunities to experiment in non-visible portions of the electromagnetic spectrum. With optics, we found that talking about everyday objects from a physics perspective resulted in thoughtful and challenging questions, such as, "When you look in a mirror, you see yourself. Is it possible to actually see a mirror?" In modern physics and astronomy, investigations necessarily relied upon modeling, including computer simulations, a coin-flipping exercise to demonstrate radioactive decay rates, and a stretched rubber sheet to represent the warping of space-time around massive objects.

Some concepts of modern physics generated stimulating discussions because of their relationship with the monastics' understanding of the nature of the universe and the Buddhist idea of causality. Nuclear decay and the Big Bang theory are examples. In nuclear decay, a statistical understanding of when a nucleus might decay agrees with the Buddhist idea of "very hidden phenomena" that cannot be directly perceived. We also found that the monastic scholars had much less trouble than our typical undergraduate students with many of the concepts from relativity. In Buddhist philosophy, ultimate nature is called "emptiness," or the lack of absoluteness, which is consistent with the lack of absolute length and time in the theory of relativity. Overall, many scholars seemed excited to have reached the point where the ideas of quantum physics and relativity were introduced, and particularly enjoyed trying to integrate these new concepts with their previous knowledge and experience.

One pedagogical technique that did not work as anticipated was introductory motivational questions. To pique interest in and generate some preliminary ideas, a question such as "What do you think causes sunspots?" might be posed. More often than not, these questions were so compelling that scholars tried to explore all the possible answers and moving scholars back to the lecture at hand felt like a disservice to their curiosity. Eventually, the most interesting questions were culled in favor of smaller, bite-sized questions that were carefully placed where they could be easily answered based upon previously presented information. 
Something is undoubtedly lost with this modification, but we found it necessary to make this sacrifice in order to complete the curriculum.

\section{SUMMARY AND DISCUSSION}

There were two main takeaways from teaching the ETSI physics curriculum to these scholars. High-engagement pedagogy worked well, based on feedback we received from the experienced translators and from scholars who attended multiple years of classes. Since we could not use the "crutch" of mathematical equations, we had to rely on demonstrations, hands-on experimentation, and active discussion/debates, which is commensurate with the way Buddhist philosophy is taught. In Buddhism, all scholars work on three main areas: the base, the path, and the result. The base is hands-on reality, whereby demonstrations and experiments facilitate the path towards enlightenment. By construction, this pedagogy engaged the scholars, which we observed to stimulate their appetite for understanding.

Secondly, translation was a challenge, broadly speaking. Many physics terms were only newly translated into Tibetan, and some chosen translations had linguistic baggage (e.g., gate vs switch). Some physics concepts were unfamiliar to some translators, and so this was a challenge for simultaneous translation. Nevertheless, due to their monastic training, the scholars tended to ask many questions, and so most translation issues were addressed. Some questions that undergraduate students might have hesitated to ask, for fear of seeming elementary, nonetheless prompted rich discussions and important clarifications with monastic scholars. Other questions were deep and sophisticated, trying to probe at the nature of reality itself. Regardless, the scholars took ownership of their own understanding, and the teacher was there to be a guide, not to profess.

Based on these qualitative observations, we suggest that incorporating keystone Buddhist traditions, such as doubting core concepts and debating, would benefit undergraduate classrooms in the United States, which too often rely on assimilation of material rather than in-depth discussions. Teaching physics conceptually to Buddhist scholars provided us with a vivid reminder of the importance of connecting with students at the cultural level and

\section{REFERENCES}

Aikenhead, G. S., and Jegede, O. J. (1999). Cross-Cultural Science Education: A Cognitive Explanation of a Cultural Phenomenon. J. Res. Sci. Teach. 36, 269-287. doi:10.1002/(sici)1098-2736(199903)36:3<269:aid-tea3>3.0.co;2-t

Chabay, R. W., and Sherwood, B. A. (2018). "Matter and Interactions," in Electric and Magnetic Interactions. 4th Edition (Hoboken, NJ: Wiley), 2.

Dreyfus, G. B. (2003). The Sound of Two Hands Clapping: The Education of a Tibetan Buddhist Monk. Oakland, CA: University of California Press.

[Dataset] ETSI (2021). Emory-Tibet Science Initiative. ETSI 13, 2021, 2021 . [Online].

French, R. S., and Prather, E. (2018). "Uncovering the Unknown Unknowns of Peer Instruction Questions," in Physics Education Research Conference 2018, Washington, DC, August 1-2, 2018 (PER Conference). linking the students' lived experience with physics concepts. This too applies in the United States, recognizing that students within our society come to the physics classroom with unique sets of beliefs and experiences.

\section{DATA AVAILABILITY STATEMENT}

The original contributions presented in the study are included in the article/Supplementary Material, further inquiries can be directed to the corresponding author.

\section{ETHICS STATEMENT}

Written informed consent was obtained from the individual(s) for the publication of any potentially identifiable images or data included in this article.

\section{AUTHOR CONTRIBUTIONS}

$\mathrm{JBe}, \mathrm{JBu}, \mathrm{MaK}, \mathrm{MiK}, \mathrm{AL}, \mathrm{JZ}$, and $\mathrm{KN}$ all equally contributed to the writing of the article based on their experiences in the ETSI program. TT provided crucial oral and written insight during the program and during the writing process.

\section{FUNDING}

ETSI is supported in part by a grant from the John Templeton Foundation.

\section{ACKNOWLEDGMENTS}

We acknowledge generous support from Emory University and the ETSI program that made this experience possible. We also acknowledge informal discussions with all of the ETSI participating faculty and monastic scholars, for whom we owe a debt of gratitude.

Hake, R. R. (1998). Interactive-Engagement Versus Traditional Methods: A Six Thousand-Student Survey of Mechanics Test Data for Introductory Physics Courses. Am. J. Phys. 66, 64-74. doi:10.1119/1.18809

Hewitt, P. G. (2010). Conceptual Physics. Boston, MA: Pearson Education, Limited. Novak, G., Patterson, E., Gavrin, A., and Christian, W. (1999). Just-in Time Teaching. Upper Saddle River, NJ: Prentice Hall.

Kalupahana, D. J. (1975). Causality: The Central Philosophy of Buddhism. Honolulu, HI: University Press of Hawaii.

Mazur, E. (1997). "Peer Instruction: A User's Manual," in Series in Educational Innovation (Upper Saddle River, NJ: Prentice-Hall). doi:10.1063/1.881735

Meltzer, D. E., and Thornton, R. K. (2012). Resource Letter ALIP-1: ActiveLearning Instruction in Physics. Am. J. Phys. 80, 478-496. doi:10.1119/ 1.3678299

Redish, E. F. (2003). Teaching Physics with the Physics Suite. Hoboken, NJ: John Wiley \& Sons. 
Redish, E. F. (2012). "The Role of Context and Culture in Teaching Physics: The Implication of Disciplinary Differences," in Keynote address presented at the World Conference on Physics Education 2012, Istanbul, Turkey, July 1-6, 2012 (Baheçeşehir University). Available at: https://arxiv.org/abs/1207.4840.

Conflict of Interest: The authors declare that the research was conducted in the absence of any commercial or financial relationships that could be construed as a potential conflict of interest.

Publisher's Note: All claims expressed in this article are solely those of the authors and do not necessarily represent those of their affiliated organizations, or those of the publisher, the editors, and the reviewers. Any product that may be evaluated in this article, or claim that may be made by its manufacturer, is not guaranteed or endorsed by the publisher.

Copyright (c) 2021 Beck, Burton, Kamela, Kuchera, Lovell, Nordstrom, Tenzin and Ziffer. This is an open-access article distributed under the terms of the Creative Commons Attribution License (CC BY). The use, distribution or reproduction in other forums is permitted, provided the original author(s) and the copyright owner(s) are credited and that the original publication in this journal is cited, in accordance with accepted academic practice. No use, distribution or reproduction is permitted which does not comply with these terms. 